\title{
Head-on collision of a large vortex ring with a free surface
}

\author{
Museok Song, ${ }^{\text {a) Luis P. Bernal, }}{ }^{\text {b) }}$ and Grétar Tryggvason ${ }^{c)}$ \\ College of Engineering, University of Michigan, Ann Arbor, Michigan 48109
}

(Received 6 May 1991; accepted 11 March 1992)

\begin{abstract}
The generation of free surface waves due to the head-on collision of a vortex ring with the free surface is studied experimentally and numerically. The interaction process is investigated by looking at the free surface evolution with the aid of a shadowgraph image, by measurement of the free surface elevation, and by visualizing the vortex ring with hydrogen bubbles. Three different free surface patterns are observed, depending on the strength of the vortex ring: (1) A single circular depression of the free surface for weak vortex rings and at very early times for strong vortex rings, (2) axisymmetric radially propagating waves in the early stage of the interaction of strong vortex rings with the surface, and (3) fully three-dimensional waves that depend on the local structure of the vortex core. Numerical simulations are used to address the observed axisymmetric phenomena.
\end{abstract}

\section{INTRODUCTION}

The free surface signature of ship wakes has recently been the subject of considerable interest motivated primarily by observations using remote sensing devices. Large turbulent wakes generally cause surface deformations of many length scales. While the large-scale structures of the wake may be of considerable interest, it is the short waves that lead to the signal detected by remote sensing. The question of how short waves are generated and modified by a large-scale motion therefore plays a fundamental role in the studies of signatures of ship wakes. Here, we report on an experimental and computational investigation of a large vortex ring colliding head-on with the surface. The motivation comes from the observation that large upwelling regions appear frequently on the surface above a turbulent flow, and these upwelling regions seem to be a major source of short surface waves.

Experiments on the interaction of vortices with a free surface are not new. Observations of the signature of vortex pairs, both trailing vortices of moving wings (Sarpkaya ${ }^{1}$ and Hirsa $^{2}$ ) as well as more two-dimensional pairs generated by moving flaps (Willmarth et al. ${ }^{3}$ ) show two basic types of deformation at the surface: striation perpendicular to the axis of the vortices and scars parallel to the vortices. Both marks are directly related to vortices near the surface, the scars are due to the primary vortices, and the striations are the result of intensification of secondary vorticity. Computational studies include Willmarth et al., ${ }^{3}$ Sarpkaya et al., ${ }^{4}$ Telste, ${ }^{5}$ Yu and Tryggvason, ${ }^{6}$ and Dommermuth and Yue. ${ }^{7}$ Although significant surface deformation can take place as the vortices collide with the surface, relatively little generation of waves-that propagate away from the interaction region-appears to have taken place in the experiments reported in the literature. Experimental studies of head-on collisions of a vortex ring

\footnotetext{
a) Present address: Department of Naval Architecture, College of Science and Technology, Hongik University, Chochiwon-Eup, Younki-Gun, Chungcheong-Nam Do, South Korea.

b) Department of Aerospace Engineering.

c) Department of Mechanical Engineering and Applied Mechanics.
}

with a free surface were also conducted by Cerra and $\mathrm{Smith}^{8}$ who investigated the evolution of the ring and compared it with the results of a collision with a solid surface. Dahm et al. ${ }^{9}$ reported a head-on collision of a vortex ring with a weak density interface.

Experiments on more complex flow configurations were conducted by Bernal and Madnia ${ }^{10}$ who used a jet located slightly below the free surface. Contrary to the vortex pairs, this flow generated a considerable amount of short waves. Bernal and Madnia ${ }^{10}$ noticed that the point where the jet first encounters the surface is where significant wave generation takes place, and identified the "opening up," or reconnection with the surface of vortex rings as the leading generation mechanism of short waves. Bernal and $\mathrm{K}$ won, ${ }^{11}$ and $\mathrm{K}$ won, ${ }^{12}$ experimentally investigated this mechanism in more detail, focusing on the collision of a small laminar vortex ring approaching the free surface at various angles. They found that the rings reconnected with the surface for a wide range of approach angles, forming a $\mathrm{U}$ vortex that propagated in the direction of the original ring. For rings within a somewhat narrow range of approach angles, the $U$ vortex subsequently breaks into two $U$ vortices when the bottom of the original $U$ collided with the surface and "opened up." The reconnection took place very rapidly compared with the bulk motion of the vortex ring. Although considerable surface deformation took place both before and after reconnection, it was the reconnection process itself that produced surface waves that propagated radially outward from the spot where reconnection took place. The results correlated well with what was seen in the jet experiment. However, the jet was at low to moderate Reynolds numbers, and the vortex ring experiments were also conducted at low Reynolds numbers. In ship wakes, the Reynolds number is generally very high, and the vortical structures generating the surface signature are large and turbulent. The experiments of Hirsa ${ }^{2}$ on vortex pairs were done at relatively high Reynolds numbers, but even when the pairs became turbulent little wave generation was observed.

In the present study, vortex rings that are both larger and at higher Reynolds numbers than previously tested are 


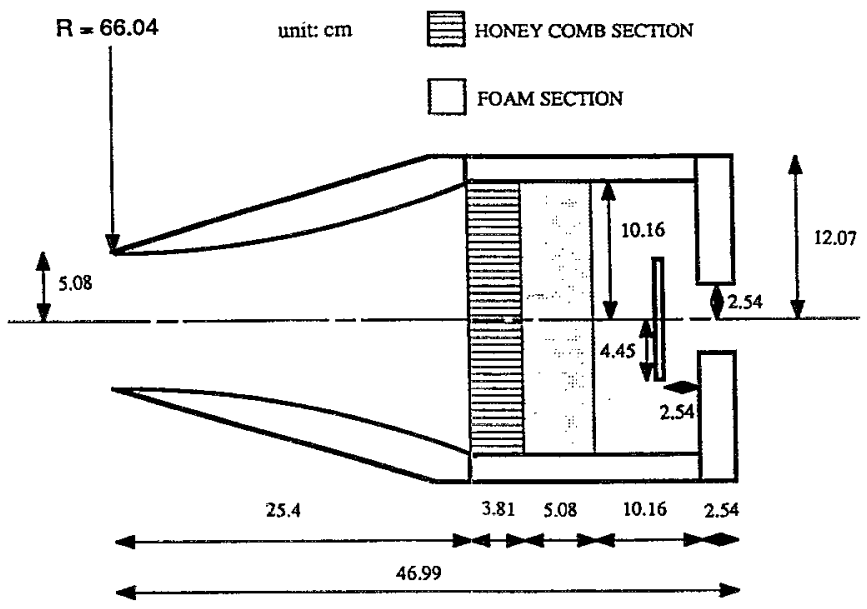

FIG. 1. Vortex ring generator. All dimensions are in centimeters.

used in an exploratory investigation of the signature of a large upwelling region. The primary purpose is to identify the basic mechanism of the interaction process. The wide range of scales ranging from the size of the ring down to the length of capillary waves on the surface, makes full numerical simulations impossible. It is therefore necessary to break the problem into subproblems of manageable size. The experiment allows the identification of such more manageable topics. An axisymmetric vortex filament model is then proposed as a sufficient representation of the early stage of the evolution, before substantial threedimensionality sets in. The model is verified by quantitative comparisons with experimental results.

Preliminary results were presented at the 18th Symposium on Naval Hydrodynamics in Ann Arbor, Michigan on 20-24 August 1991 (see Song et al. ${ }^{13}$ ).

\section{EXPERIMENTAL MEASUREMENTS AND NUMERICAL SIMULATIONS}

\section{A. Flow facility and instrumentation}

The experiments were conducted in the tow tank facility at the Ship Hydrodynamics Laboratory of the University of Michigan which has a tow basin that is $3 \mathrm{~m}$ deep, 6 $\mathrm{m}$ wide, and $100 \mathrm{~m}$ long, large enough to accommodate a large vortex ring. The vortex generator was constructed based on the design of Kwon, ${ }^{12}$ and is shown along with the associated plumbing in Figs. 1 and 2. The generator consists of a settling section and a contraction section. The settling section contains a flow diverter, a foam section, and a honeycomb section. As shown in Fig. 1, the contraction section is of a circular arc shape and has an exit diameter $\left(D_{0}\right)$ of $10.16 \mathrm{~cm}$. The vortex generator was positioned approximately six exit diameters from the water surface using a rigid frame attached to the bottom of the tow basin.

The rings were produced by a brief outflow of water through the generator, driven by a pressurized water tank. The duration of the water pulse was controlled by a solenoid valve. The characteristics of the vortex ring formed depend on the pressure in the reservoir tank and the dura-

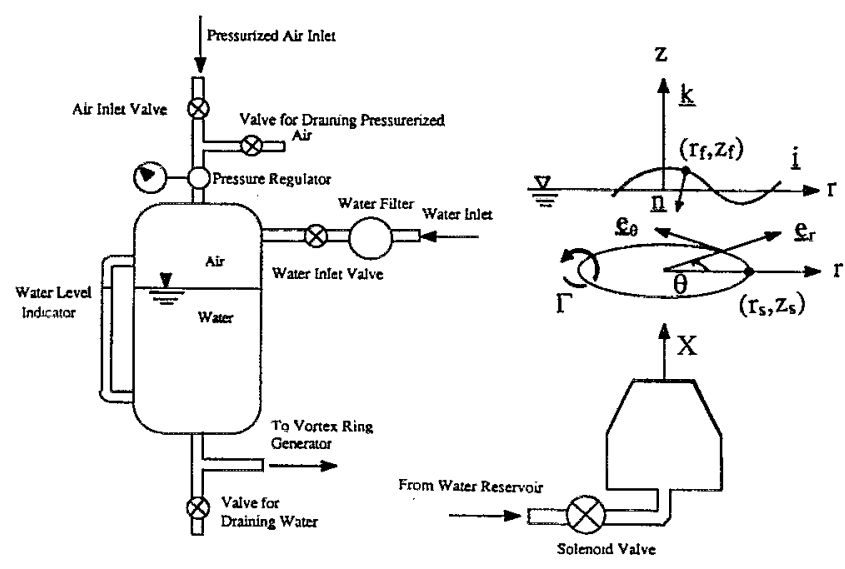

FIG. 2. Schematic arrangement of the experimental apparatus and coordinate system.

tion of the water pulse. A high reservoir pressure results in a high momentum through the generator and consequently high exit velocity and a large vortex ring circulation. A longer pulse duration also results in increased circulation of the ring but the size of the boundary layer deposited in the flow-which one would expect to determine the core size-increases as the pulse duration increases while it decreases as the exit velocity is increased. Both the circulation and the core size have an effect on the propagation speed of the resulting vortex ring. A detailed discussion of the characteristics of vortex rings as they relate to the generator operating parameters is given by Kwon, ${ }^{12}$ Didden, ${ }^{14}$ and Glezer. ${ }^{15}$

To characterize the vortex rings, we measured the fluid velocity, $u(t)$, versus time with a hot film anemometer on the axis of the ring motion, at 3.0 and 3.375 diameters above the generator exit [where the ring was well developed $\left.\left(K_{\text {won }}{ }^{12}\right)\right]$. The evolution of the vortex ring was also videotaped with an underwater camcorder. This velocity was used to determine the circulation $(\Gamma)$ and the propagation speed of the ring $(U)$. We used the time for $u(t)$ to reach its maximum at two different locations to determine the propagation speed of the ring. To determine the circulation of the vortex ring, we used

$$
\Gamma=2 u_{\max } R \text {. }
$$

This formula relates the maximum centerline velocity to the size and strength of a single vortex filament ring without core. Here, $R$ is the radius of the vortex ring measured from the videotape. In a few cases we have compared this value with a more accurate estimate given by

$$
\Gamma=\oint \mathbf{u} \cdot d \mathbf{l} \approx U \int_{0}^{\infty} u(t) d t,
$$

and found close agreement [circulation obtained using Eq. (1) is generally $10 \%$ lower than that of Eq. (2), see $\mathrm{Kwon}{ }^{12}$ ] as long as there was no significant jetlike tail behind the ring. The radius of the vortex core $e$ was estimated from the measured values of $\Gamma, R$, and $U$, using the theoretical result for the propagation speed of a vortex ring with a constant vorticity core $\left(\operatorname{Lamb}^{16}\right)$, 


$$
U=\frac{\Gamma}{4 \pi R}\left(\ln \frac{8 R}{e}-\frac{1}{4}\right)
$$

Two synchronized cameras were used to visualize the flows: a side view to follow the vortex ring evolution and a top view for the free surface deformation. To observe the ring, hydrogen bubbles were generated near the exit of the vortex generator. The motion was recorded on a sequence of still pictures or on video. The generation time of the hydrogen bubbles was kept as short as possible in order to minimize the effects of the hydrogen bubbles on the flow. To capture the free surface deformation a shadowgraph technique was used. A single $150 \mathrm{~W}$ bulb was located well below the free surface and a semitransparent plastic screen $(120 \times 120 \mathrm{~cm})$ was positioned $3 \mathrm{~cm}$ above the free surface. These free surface images were also recorded with a still picture camera or a camcorder.

To quantify the free surface deformation, we measured the time history of the free surface elevation at several locations along the radial direction on the surface. The measurements were done with a capacitance probe, $2 \mathrm{~mm}$ in diameter and $10 \mathrm{~cm}$ long. This probe is very similar to the one used by Henderson and Hammack, ${ }^{17}$ except that the diameter of our probe is slightly larger. Through a dynamic calibration it was verified that the troughs and crests were captured with acceptable accuracy.

In presenting our results nondimensional variables are used. We nondimensionalize lengths by $R$, time by $R^{2} / \Gamma$, velocity by $\Gamma / R$, vorticity by $\Gamma / R^{2}$, and surface tension by $\rho \Gamma^{2} / R$. This gives the controlling parameters as the Froude number $\mathrm{Fr}=\Gamma / \sqrt{g R^{3}}$, the Reynolds number $\operatorname{Re}$ $=\Gamma / v$, and the Weber number $\mathrm{We}=\rho \Gamma^{2} / \sigma R$. Here $g$ is the acceleration due to gravity, $v$ is the kinematic viscosity, and $\sigma$ is the surface tension.
Surfactants are known to be of considerable significance in many vortex/free surface problems, causing the generation of secondary vortices (Bernal et $a l .^{18}$ ). Here, the surface was cleaned by a surface current diverging away from the test region during the night and, as a result, we did not observe the formation of secondary vortices due to surface contaminant. While large surface deformation can also cause secondary vorticity generation, little, or no secondary vorticity is generated when surface deformations are small, as is the case here.

\section{B. Numerical method}

For the numerical simulations we use a vortex/ boundary integral method. The flow is assumed to be axisymmetric, inviscid, incompressible, and irrotational except for the ring. If the velocity potential on the free surface and the geometry of the free surface are known, the tangential velocity at the surface can be evaluated by differentiation along the surface. The normal velocity on the free surface can then be found by solving

$$
\begin{aligned}
2 \pi(\mathbf{n} \cdot \mathbf{u})_{f}= & \mathbf{n}_{f}\left[\mathscr { f } _ { \partial \Omega } \left((\mathbf{n} \cdot \mathbf{u}) \frac{\left(\mathbf{x}_{f}-\mathbf{x}_{s}\right)}{\left|\mathbf{x}_{f}-\mathbf{x}_{s}\right|^{3}}\right.\right. \\
& \left.+(\mathbf{n} \times \mathbf{u}) \times \frac{\left(\mathbf{x}_{f}-\mathbf{x}_{s}\right)}{\left|\mathbf{x}_{f}-\mathbf{x}_{s}\right|^{3}}\right) d S_{s} \\
& \left.+\int_{\Omega_{\omega}}\left(\omega \times \frac{\left(\mathbf{x}_{f}-\mathbf{x}_{s}\right)}{\left|\mathbf{x}_{f}-\mathbf{x}_{s}\right|^{3}}\right) d V_{s}\right],
\end{aligned}
$$

where indicates a principal value integral (see, e.g., Brockett et al. $\left.{ }^{19}\right)$. This is a Fredholm integral equation of the second kind and easily solved by iteration. In our axisymmetric case, Eq. (4) becomes

$$
\begin{aligned}
\frac{\pi}{2}(\mathbf{n} \cdot \mathbf{u})_{f}= & \mathbf{n}_{f}\left(\oint_{0}^{\infty}(\mathbf{n} \cdot \mathbf{u})_{s} r_{s}\left\{\left[\mathbf{i}\left(r_{f}-r_{s}\right)+\mathbf{k}\left(z_{f}-z_{s}\right)\right] I_{1}+2 \mathbf{i} r_{s} I_{2}\right\} d r_{s}+\oint_{0}^{\infty}(\mathbf{n} \times \mathbf{u}) r_{s}\left\{\left[\mathbf{i}\left(z_{f}-z_{s}\right)+\mathbf{k}\left(r_{s}-r_{f}\right)\right] I_{1}+\left[2 \mathbf{k} r_{f}\right.\right.\right. \\
& \left.\left.\left.-2 \mathbf{i}\left(z_{f}-z_{s}\right)\right] I_{2}\right\} d r_{s}+\Gamma r_{s}\left\{\left[\mathbf{i}\left(z_{f}-z_{s}\right)+\mathbf{k}\left(r_{s}-r_{f}\right)\right] I_{1}+\left[2 \mathbf{k} r_{f}-2 \mathbf{i}\left(z_{f}-z_{s}\right)\right] I_{2}\right\}\right)
\end{aligned}
$$

Here, $r, z, \mathbf{i}$, and $\mathbf{k}$ are space variables and unit vectors shown in Fig. 2. Subscripts $f$ and $s$ represent field and singular points, respectively, $\Gamma$ is the circulation of the vortex ring, and

$$
\begin{aligned}
& I_{1}=A\left[E(B) /\left(1-B^{2}\right)\right], \\
& I_{2}=I_{1}-A\left\{\left[E(B) /\left(1-B^{2}\right) B^{2}\right]-\left[K(B) / B^{2}\right]\right\},
\end{aligned}
$$

where $K$ and $E$ are elliptic integrals of first and second kind, respectively, $A=\left(a^{2}+b^{2}\right)^{-1.5}, B=\sqrt{2 b^{2} /\left(a^{2}+b^{2}\right)}$, $a^{2}=r_{f}^{2}+r_{s}^{2}+\left(z_{f}-z^{s}\right)^{2}$, and $b^{2}=2 r_{f^{\prime}} r_{s}$. In the numerical implementation, $a^{2}$ is replaced by $a_{\delta}^{2}=a^{2}+\delta^{2}$. This models the finite core of the vortex filament $\left(\right.$ Leonard $\left.{ }^{20}\right)$. After solving for $n \cdot u$, the velocity potential on the surface is updated by Bernoulli's equation,

$$
\frac{d \phi}{d t}=\frac{1}{2} \mathbf{u} \cdot \mathbf{u}-\frac{z}{\mathrm{Fr}^{2}}+\frac{1}{\mathrm{We}}\left(\frac{\partial^{2} z}{\partial r^{2}}+\frac{1}{r} \frac{\partial z}{\partial r}\right),
$$

and the kinematic relation,

$$
\frac{d \mathbf{x}}{d t}=\mathbf{u},
$$

is used to update the position of the free surface and the vortex ring. Notice that the surface tension is linearized in Eq. (8) since the Weber number is large for the cases tested (order of 100) and surface deformations are expected to be small. To complete the formulation we impose $\phi=\nabla \phi=0$ as $|x| \rightarrow \infty$ and take $\phi=0$ at $z=0$ as initial conditions. Tests using many filaments $(7,19,37$, and 61$)$ 
TABLE I. Characteristics of vortex rings. Here $P^{*}$ is the gauge pressure in the tank; $T_{0}$ is the time during which the valve is open; $R, U$, and $u_{\max }$ are the measured vortex ring radius, propagation velocity, and the maximum centerline velocity, respectively; $\Gamma$ is the circulation of the ring estimated by Eq. (1); Re and Fr are the Reynolds and Froude number defined in the text. The ratio of the ring radius to the estimated core radius [Eq. (3)] is denoted by $R / e$ and $L / D_{0}$ is the "aspect" ratio of the ejected slug ( $D_{0}$ is the diameter of the mouth of the vortex ring generator and $L$ is the length of the slug, thus, $\pi L D_{0}^{2} / 4=$ volume of water ejected).

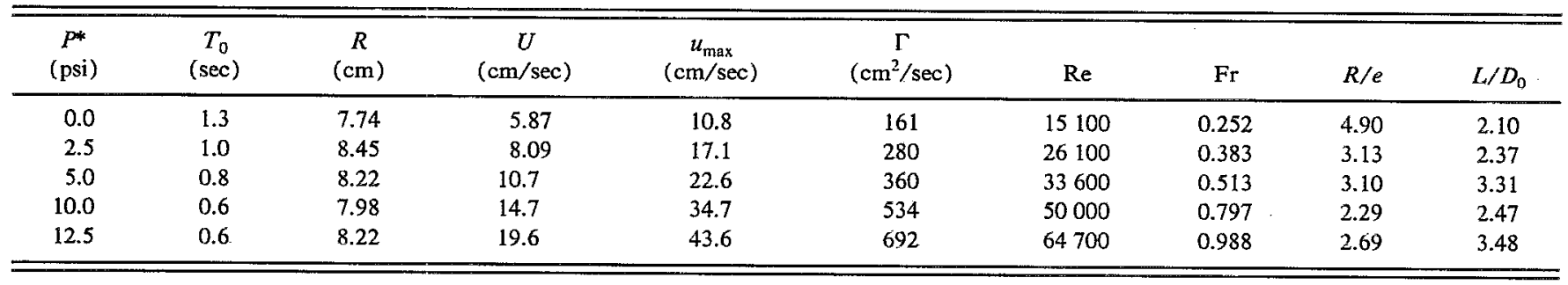

to model the ring suggest that a single filament with a correctly selected core parameter $\delta$ gives a sufficiently good representation of the ring. As the filament stretches, $\delta$ is varied to keep the volume of the core constant. The singular integrals on the free surface are evaluated with a smoothing parameter (replacing $a$ by $a_{\delta}$ ), carefully chosen from convergence tests. The free surface has to be truncated at a finite distance from the ring. To allow long time simulation, a "sponge" layer is imposed at the edge of the numerical domain to damp outgoing waves. The velocity potential and the surface elevation were multiplied after each time integration by a function $\exp \left[-(r / 27.0)^{30}\right]$ where the calculation domain was truncated at $r=30.0$. A high-order predictor-corrector with variable step size is used for the time integration.

\section{RESULTS AND DISCUSSION}

Five vortex rings were selected from preliminary experiments for a more detailed study. Table I shows the characteristics of these rings. The ranges of $\mathrm{Re}$ and $\mathrm{Fr}$ are $15000-65000$ and $0.25-1.0$, respectively. In what follows, we discuss the results for the highest and the lowest Froude number in detail. Figure 3 shows a time sequence of the vortex ring for $\mathrm{Fr}=0.252$ and $\mathrm{Re}=15100$, seen from the side. The image above the free surface is due to reflection in the free surface and the tail near the ring axis is formed by hydrogen bubbles not captured by the ring. The first frame when the dimensionless time $T$ is 0.8 , shows the vortex ring while it is still far away from the free surface. The dimensionless time is set to zero when the rings are 2.5 ring radii away from the free surface. There are small wavy disturbances along the core, but the flow is regular, the core is clearly visible, and the Kelvin oval-bounding the fluid carried with the vortex ring-is also quite smooth. The smoothness of the ring is in sharp contrast with the images of turbulent rings reported by Glezer. ${ }^{15}$ The second and third frames show that the vortex ring expands outward as it approaches the free surface. In the third frame we see, in addition to the expansion, a deformation of the core indicating an instability. In the fourth and fifth frames, the ring has expanded further but the thin vortex core is not continuous around the circumference of the ring. To the extent that the bubbles trace the vortex core, it appears that the core reconnects locally to the free surface. The velocity measurement on the ring axis, Fig. 4, indicates that the flow field induced by the vortex ring, at least near the propagation axis, is laminar.

Shadowgraph images of the free surface, corresponding to the side view pictures in Fig. 3, are given in Fig. 5. At the beginning of the interaction, a single circular dark band, indicating a depressed free surface, is observed. The radial location of this dark band is just outside of the vortex core and moves outward as the vortex ring expands. When $T$ is 16.1 (second frame), nonaxisymmetric features

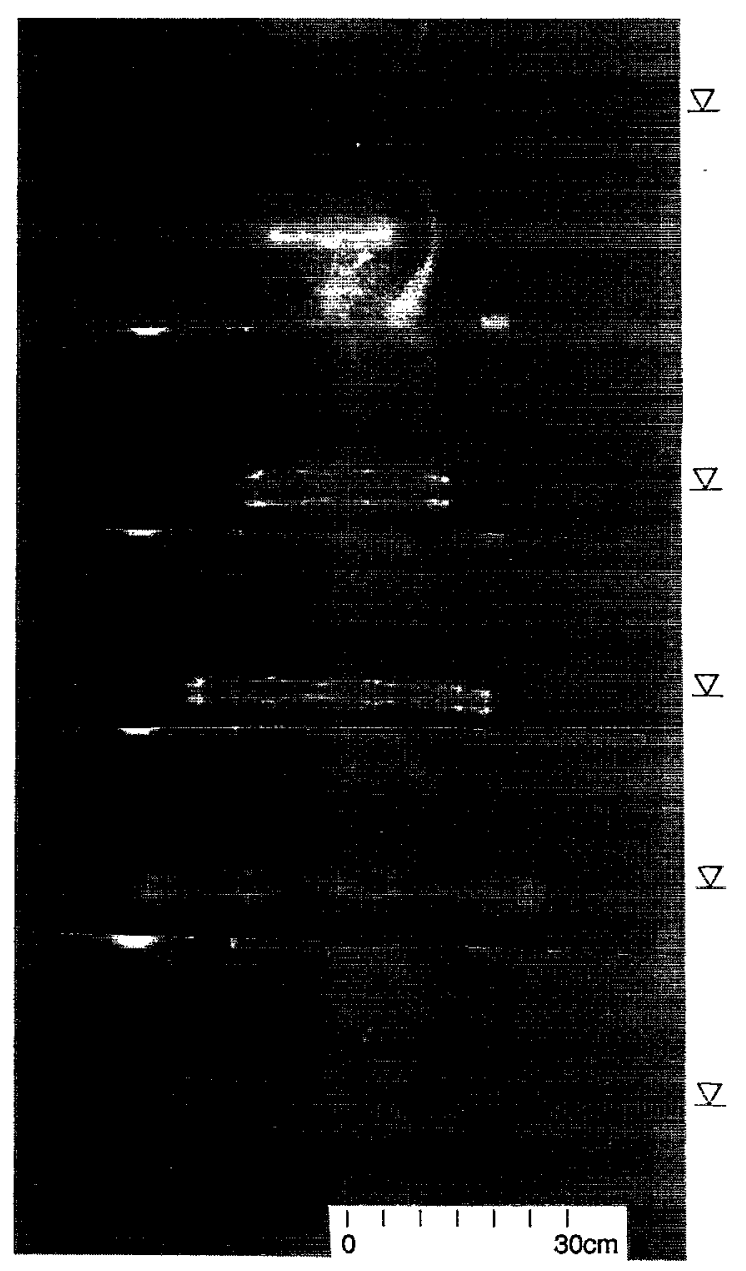

FIG. 3. Side views of the vortex ring evolution. $\mathrm{Fr}=0.252 ; T=0.811$, $12.6,16.1,19.3$, and 21.9 (from top to bottom); $\underline{\nabla}$ is the approximate location of the free surface 


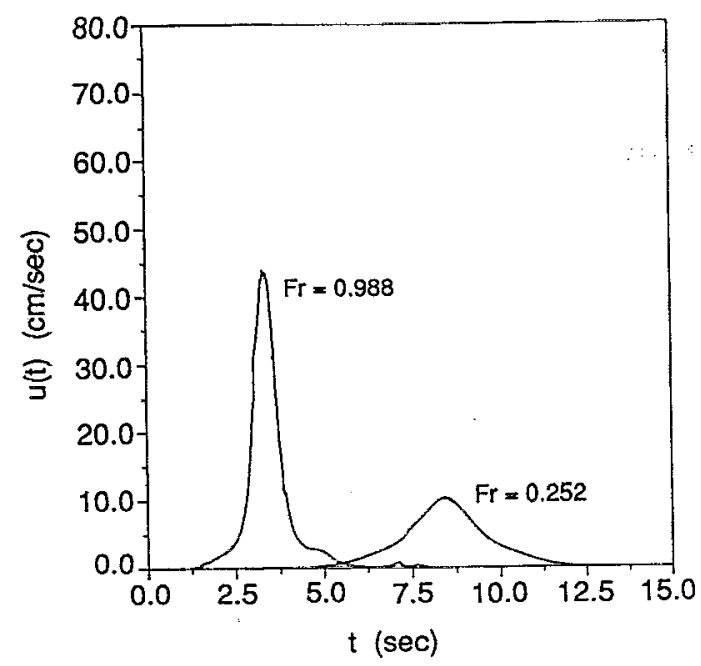

FIG. 4. Hot film measurement of the velocity, $u(t)$, at $X / D_{0}=3.0$.

are observed on the surface in addition to the dark band. These features are weak "striations" over the dark band, i.e., lines perpendicular to the core of the vortex ring. The flow in the second picture is still predominantly axisymmetric. Considerable three-dimensionality is apparent in the third frame, at $T=19.3$. In addition to increased irregularities in the main dark band, two new features have appeared: dark spots and short waves. The dark spots are depressed free surface regions where vortex filaments terminate. The small waves are not observed before the dark spots appear on the surface and seem to be generated as the vortex filament opens up at the free surface. The last frame, at $T=21.9$, shows several dark spots, but no coherent dark band. This indicates that an azimuthally coherent vortex ring no longer exists. At this stage the flow consists of many U-shaped vortex filaments, with their "legs" termi-
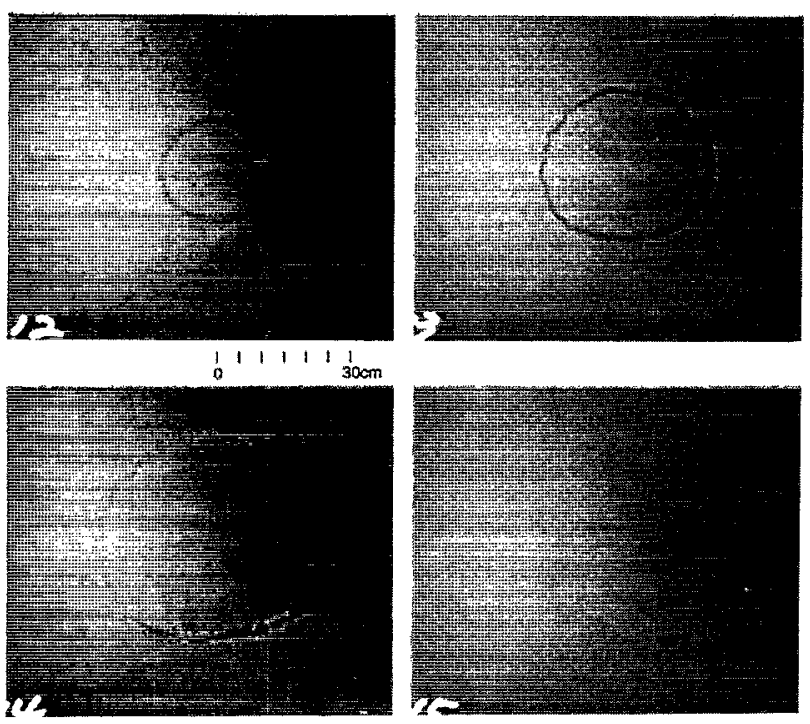

FIG. 5. Shadowgraph images of free surface signature. $\mathrm{Fr}=0.252 ; T$ $=12.6$ (upper left corner), 16.1, 19.3, and 21.9.
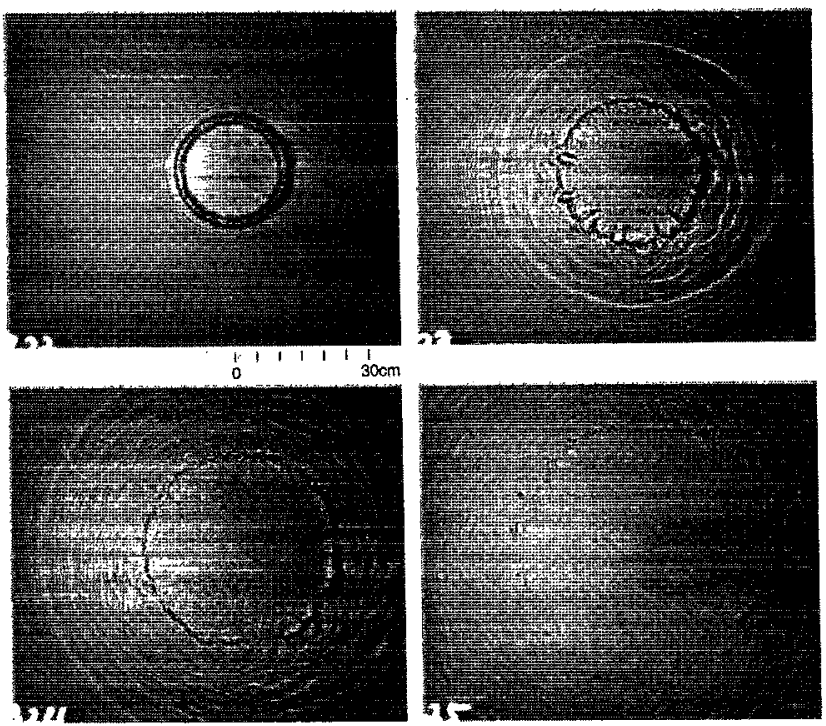

FIG. 6. Shadowgraph images of free surface signature. $\mathrm{Fr}=0.988 ; T$ $=11.5$ (upper left corner), 21.4, 33.5, and 45.5.

nating at the free surface. These $U$ vortices propagate outward due to their own self-induced velocity.

The shadowgraph images of the free surface for a larger Froude number $(\mathrm{Fr}=0.988$ and $\mathrm{Re}=64700)$, are shown in Fig. 6. This sequence shows several features that are different from the lower Froude number case shown in Fig. 5. The first frame $(T=11.5)$ shows a strong axisymmetric dark band with a bright region in it that indicates a local rise of the surface in the dark band. Careful observation reveals that this bright region consists of very dense striations perpendicular to the main vortex core. Moreover, short waves are already observed ahead of the dark band. Even though these short waves are slightly distorted near the dark band, they have axisymmetric structure, on the average. As the local bright region disappears, larger axisymmetric waves are generated which propagate outward. In the second frame, at $T=21.4$, the dark band has broken up in several places and developed fully threedimensional features. Dark spots signaling the "opening up" of vortex filaments are also observed near the dark band. Notice also that there is a clear boundary between the small-scale waves associated with the threedimensional structures and the already generated axisymmetric waves. The end of the generation of these short, globally axisymmetric waves seems to coincide with the time when significant three-dimensionality starts to appear. At later times $(T=33.5$ and 45.5$)$ dark spots and short waves become more pronounced while the azimuthally coherent dark band gradually disappears. In the last frame, the dark coherent band has disappeared, and the free surface signature is dominated by dark spots and irregular short waves originating at the spots. Thus the final signature is similar to the lower Froude number case, except that the short waves have larger amplitude.

The dark spots in the shadowgraph pictures of the surface indicate that the topology of the vortex lines has changed from an axisymmetric configuration at the begin- 


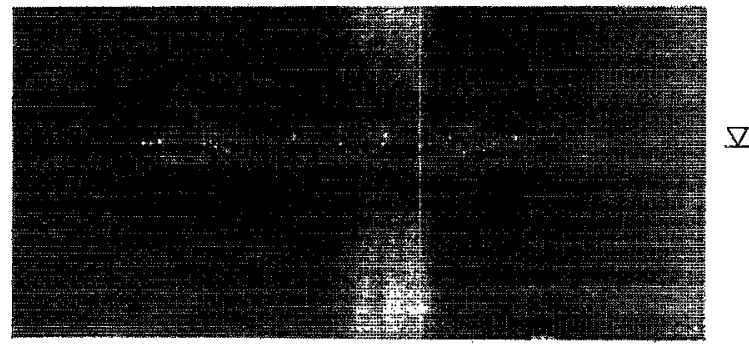

FIG. 7. Reconnected vortex filaments. $\mathrm{Fr}=0.988 ; T=45.5 ; \underline{\nabla}$ is the approximate location of the free surface.

ning of the interaction to a configuration having vortex lines attaching to the surface by a vortex reconnection process. This is clearly shown by the hydrogen bubble photograph in Fig. 7 which corresponds to the last frame in Fig. 6 . The bubbles that mark the location of the vortex core show the formation of U-shaped vortex lines as in the lower Froude number case.

The flow visualization results indicate that the vortex ring remains axisymmetric for a significant part of the interaction process, especially for the lower Fr. The simple axisymmetric flow model, presented in Sec. II B, may therefore be sufficient to describe the early evolution of the flow. In Figs. 8 and 9 we show the calculated free surface shape as a function of time. Since the amplitude of the free surface motion is relatively small, the horizontal scale is much larger than the vertical one. For $\mathrm{Fr}=0.252$, in Fig. 8 , the only noticeable free surface deformation is a single outward moving depression bounded by two convex regions. For the lager Froude number $(\mathrm{Fr}=0.988)$ in Fig. 9, the free surface above the vortex ring rises significantly due to the initial impact and the depression of the free surface outside of the vortex core is deeper. Similar depressions outboard of the vortices have also been seen in other numerical simulations, e.g., Dommermuth and $\mathrm{Yu} .^{7}$ In addition to the magnitude of the surface deformation for the different Froude numbers, the major difference in the experiments-at least initially, when the evolution is clearly axisymmetric-is the presence of short waves propagating outward from the vortex core for the strong Froude number case. Our calculations show small outward propagating waves for the larger Froude number case, whether surface tension was included or not. This wave can be seen in the last two frames in Fig. 9 and also in the last frame in Fig. 14 (to be discussed later).

The calculated and the measured vortex core trajectories are compared in Fig. 10 for $F r=0.252$ and 0.988 . The numerical results show a slight rebounding of the vortex core at the larger Froude number. This rebounding is small and is not clearly observed in the experiments. In contrast, no noticeable rebounding is observed for the lower Froude number case. These results are consistent with other investigations where surfactant effects are small (Bernal et al. ${ }^{18}$ ) and which show no rebounding of the core for low Froude numbers. The maximum closeness of the vortex core to the free surface depends on the vortex core size. At the lower

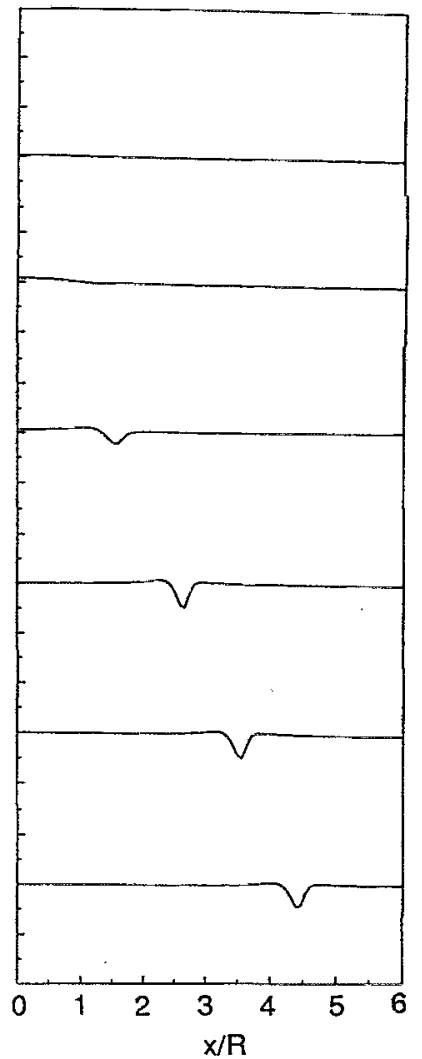

FIG. 8. Calculated free surface deformations. $F r=0.252 ; T=4.42,7.27$, $10.1,12.9,15.1$, and 17.3 (from top to bottom).

Froude number, for which the vortex core is much smaller (see Table $\mathrm{I}$ ), the core reaches closer to the free surface $(-0.201 R)$ than in the large Froude number case $(-0.375 R)$. In both cases, the numerical calculations were continued beyond the time at which the core ceases to be clearly identifiable in the experiments. Notice that the vortices do not continue to come closer to the surface, for both lower and higher Froude numbers, as vortex rings approach a flat surface would. This is because of the reaction from the deformed surface.

In Fig. 11 the locations of the vortex core in the radial and vertical directions (circles) and the radial location of the dark band (stars) are plotted versus time for $\mathrm{Fr}$ $=0.252$. The computed position of the vortex is shown by a dashed line. Only the dark band location was measured after the ring hit the free surface, since the measurement of the core location from the videotaped picture is subject to a large uncertainty at late times. The arrows indicate the time when three-dimensionality is first observed and the time when the flow becomes fully three dimensional (i.e., when the axisymmetric dark band is no longer visible). Before the vortex ring hits the free surface, when $T<8.0$, approximately, there is no surface signature and the mea-. sured and calculated vortex core trajectories agree well, even when the vortex ring is close to the free surface. As the vortex ring collides with the free surface it expands outward due to the effect of the image. The experimentally observed outward velocity is slightly lower than the com- 


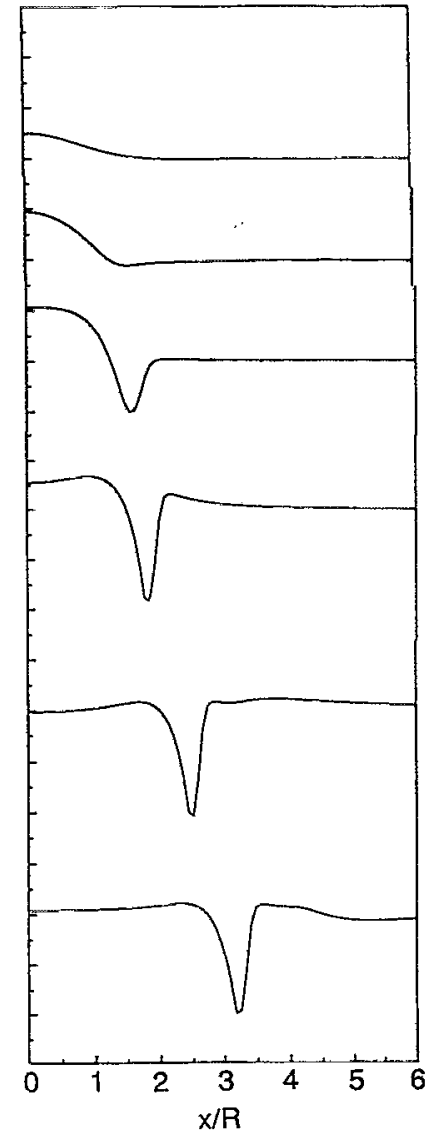

FIG. 9. Calculated free surface deformations. $\mathrm{Fr}=0.988 ; T=5.62,8.13$, $10.5,12.3,15.7$, and 19.5 (from top to bottom).

puted one, and the collision process seems to take a slightly longer time. Since the ring is observed to remain fairly coherent and axisymmetric during this stage, it is most likely that the disagreement is due to viscous effects, such as increased core size. At lower Re, it takes about $10 \mathrm{sec}$

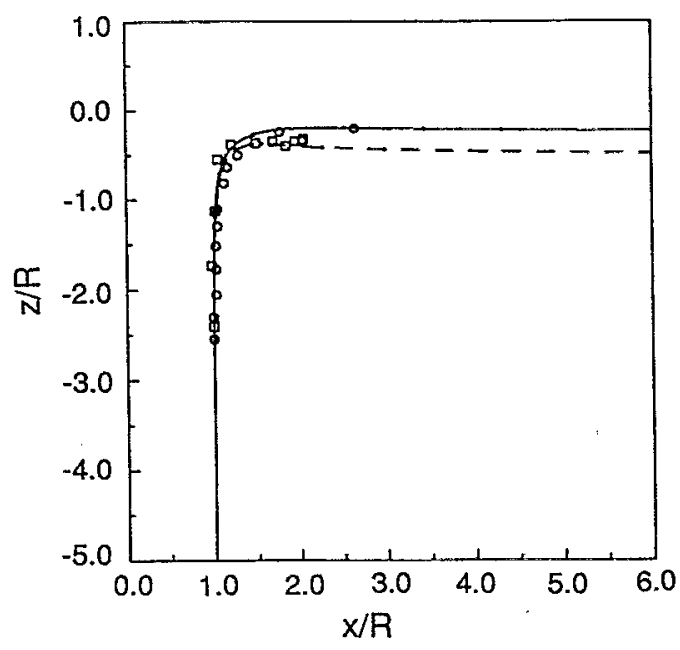

FIG. 10. Vortex core trajectory. $\mathrm{Fr}=0.252: \mathrm{O}$, experiment; - , calculation. Dots at $T=4.42,7.27,10.1,12.9,15.1$, and $17.3 \mathrm{Fr}=0.988: \square$, experiment; - - , calculation. Dots at $T=5.62,8.13,10.5,12.3,15.7$, and 19.5 .

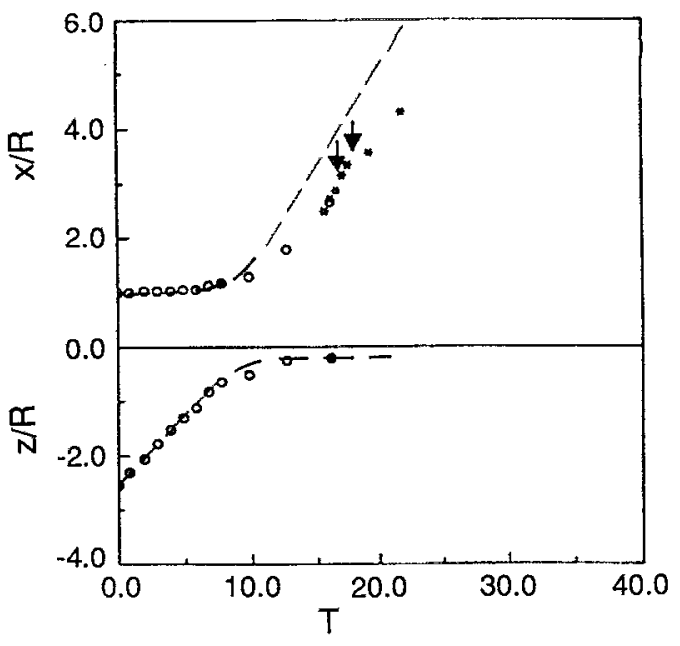

FIG. 11. Location of the vortex core (O) and dark band $(*)$ versus time from the experiment; ---, calculated location of the vortex core; $\mathrm{Fr}$ $=0.252$.

for the ring to reach the surface, while it takes about $2 \mathrm{sec}$ for the stronger rings. Therefore, not only is the Reynolds number lower but viscosity also has more time to act. For a discussion of viscous effects on high Reynolds number vortex rings see Saffman ${ }^{21}$ and Maxworthy. ${ }^{22}$ Notice the fast development of the three-dimensionality. Once threedimensionality sets in (first arrow), it does not take long for the flow to become fully three dimensional (second arrow). As a result of the development of threedimensionality the outward velocity of the ring is greatly reduced and since the numerical simulation was intended to capture only the axisymmetric aspects, comparison after the second arrow shows a large discrepancy.

Figure 12 show the same information for $\mathrm{Fr}=0.988$ As in Fig. 11 the asterisks indicate the radial location of the dark band or the dark spots. The first arrow indicates that three-dimensionality is observed at an earlier stage of

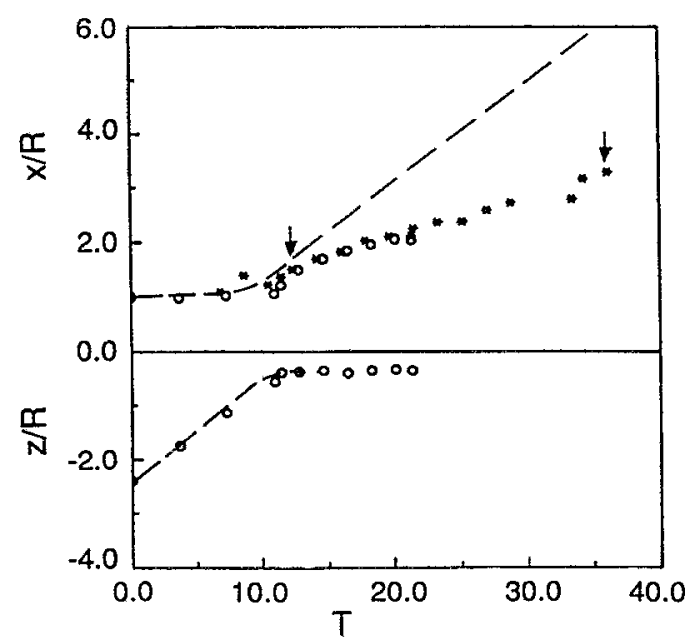

FIG. 12. Location of the vortex core $(O)$ and the dark band (*) versus time from the experiment; - - - calculated location of the vortex core; $\mathrm{Fr}=0.988$. 
(a)

(b)
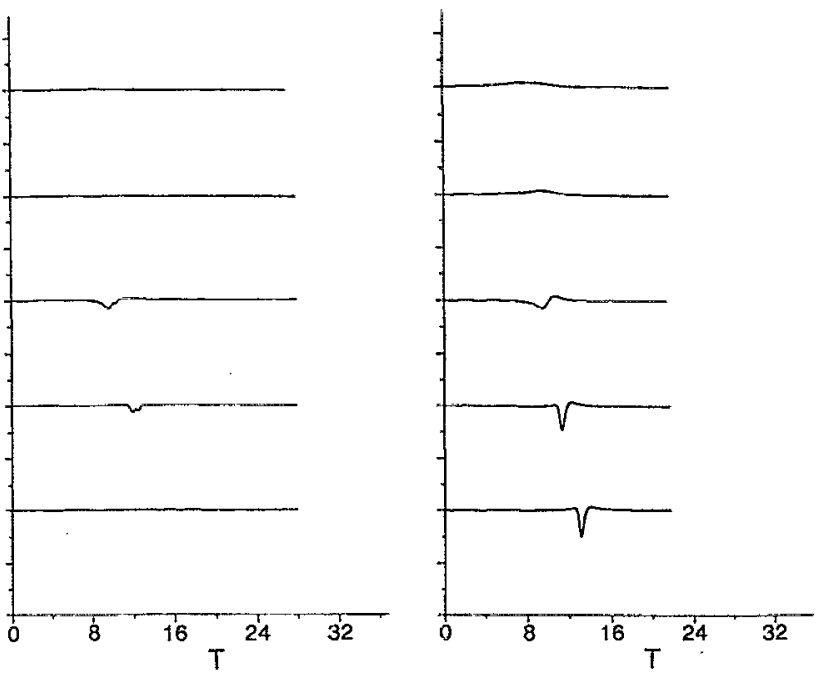

FIG. 13. Time history of the free surface elevation at fixed points for $\mathrm{Fr}=0.252$. (a) Experiment: $r / R=0.0,0.66,1.31,1.97$, and 2.63 (from top to bottom). (b) Calculation: $r / R=0.0,0.67,1.35,2.02$, and 2.70 (from top to bottom). The vertical scale is greatly enlarged.

the interaction than for the lower Fr. However, as the second arrow shows, the dark band persists relatively longer than for the lower Fr. Before the development of three-dimensionality the results of the calculation agree well with the measurements, even better than in the low Froude number case. Comparing the results of Figs. 11 and 12 , we note that the outward velocity of the core in the axisymmetric region is larger for the lower Froude number case. This is consistent with the observation that the core is closer to the surface in that case, resulting in an increased induced velocity due to the image vorticity. As in the lower Froude number case the outward propagation speed after the development of three-dimensionality is reduced significantly.

To quantify the deformation of the free surface, its elevation was measured with wave probes. The probes were located at various radial locations. The results are shown in Figs. 13 and 14 where the elevation is shown versus time for each probe. The experimental results are on the left (a), and the computed one on the right (b). For the lower Froude number ( $\mathrm{Fr}=0.252$ in Fig. 13) - as the first two frames in Fig. 5 show - there is only one smooth surface depression that passes through the probe at $r / R=1.31$ and 1.97. This deformation does not appear at $r / R=2.63$ since the flow becomes three dimensional when the dark band is at $r / R=2.5$ (see the first arrow in Fig. 11), and only the small amplitude oscillating signal is captured. The measured and calculated results are in good agreement-both magnitude and location for the probes closest to the center-although there is a small difference in the detailed shape of the signal. For $r / R>2.5$, the experimental ring has become fully three dimensional, whereas the axisymmetric model predicts a single large surface deformation. For the stronger vortex ring, Fig. 14, the free surface elevation near the center is noticeably larger than for the (a)

(b)
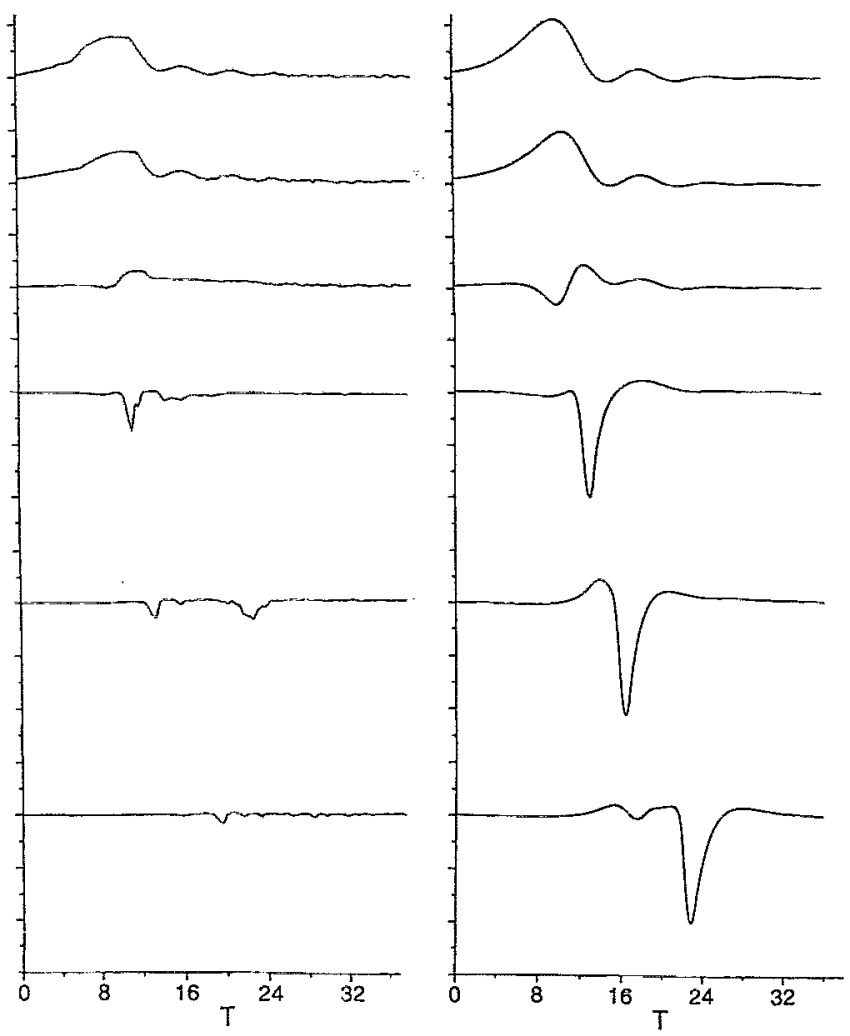

FIG. 14. Time history of the free surface elevation at fixed points for $\mathrm{Fr}=0.988$. (a) Experiment: $r / R=0.0,0.62,1.24,1.85,2.47$, and 3.71 (from top to bottom). (b) Calculation: $r / R=0.0,0.60,1.27,1.87,2.55$, and 3.82 (from top to bottom). The vertical scale is greatly enlarged.

weak vortex ring and shows smooth residual wave motion after the initial swell up of the free surface which was not captured by the shadowgraph image. Most of the signals for this stronger ring are followed by significant irregular oscillations due to the three-dimensionality that appears almost as soon as the vortex ring collides with the free surface. Comparison between the measured data and the calculation shows good agreement near the center. The calculated oscillation period of the residual wave appeared to be slightly longer than the measurement presumably due to overestimation of the time scale in the experiment [since the circulation was slightly underestimated by Eq. (1)]. Notice that the small waves that appear outside the main depression in the shadowgraph in Fig. 6 (and that were not visible in the computer surface elevation) do not show up in the wave probe data, suggesting that their amplitude is very small. (Enlarging the computed elevation in Fig. 9 shows waves of very small amplitude outside the main depression.)

The large vortex rings, used in the present investigation, are quite smooth although they are at a very high Reynolds number. Larger-scale instabilities, seen, for example, by Glezer, ${ }^{15}$ were not observed in our vortex rings, presumably because the ring diameter is very large compared to the core size. The curvature of the vortex core, which is closely related to the development of such insta- 
bilities, is small in our case. As Widnall and Sullivan ${ }^{23}$ pointed out, instability of a vortex ring is not predicted by $\Gamma / v$ alone, since the amplification of disturbances along the core depends on the quantity $(\Gamma / v)(e / R)^{4}[\ln (e / R)]^{2}$, which includes the ratio of the core size to the radius. This parameter, which seems to be the more relevant Reynolds number for the large-scale instability, is not provided by Glezer ${ }^{15}$ for his turbulent rings.

The closeness of the vortex ring to the surface is strongly dependent on the size of the core but the surface deformation influences this also. The curvature of the vortex line induces a velocity toward the surface and the core moves upward until it is a distance of the order of the core size. Then it moves outward with a velocity of the order of $\Gamma / e$ in the absence of three-dimensional instabilities. This evolution is well captured by our axisymmetric flow model where the only experimental parameter to be matched by the calculation is the size of the core.

As the vortex ring approaches the surface, the core develops an instability reminiscent of the Crow ${ }^{24}$ instability of a pair of vortex lines (see also Widnall et al..$^{25}$ and Tsai et $a l^{26}$ ) that is driven by the flow field induced by the free surface. Since the relevant length and velocity scales for this instability are $e$ and $\Gamma / e$, respectively, it seems that the appropriate Reynolds number should be $\Gamma / v$. Indeed, the instability develops earlier in the high Reynolds number cases, and more rapidly. This instability eventually results in the breaking and reconnection of the vortex lines to the free surface, leading to a significantly lower outward velocity of the vortical structure as compared to the axisymmetric state. In order to correctly describe this behavior, a different model that properly accounts for the fully threedimensional interaction needs to be developed.

\section{CONCLUSION}

The head-on collision of large vortex rings with a free surface is discussed. Experimental observations show that the rings generally have a very coherent structure until shortly after they have collided with the free surface. The core then undergoes an instability, and the ring "reconnects" with its image forming several U-shaped vortices with their legs terminating at the free surface. This reconnection process or "opening up" is the main cause for the generation of short waves. This was also found in the jet experiment of Bernal and Madnia, ${ }^{10}$ and may therefore be a generic mechanism for producing short waves during vortex/free surface interactions.

Initially, the ring and the surface signature are axisymmetric. The subsequent transition to a more threedimensional stage takes place relatively fast. The resulting short scales are much smaller than the vortex ring, but on the average the small scales have an axisymmetric distribution. The large difference between the smallest and the largest scales makes direct numerical simulations of the full problem impossible. However, the results suggest that the evolution can be divided into two phases: the initial axisymmetric phase of the large coherent ring and the transition to three-dimensionality due to the breakup of the core. Various elementary processes during each stage are accessible to numerical modeling.

We show that the first stage-until shortly after the ring has collided with the surface-is well captured by an inviscid, axisymmetric model. For lower Froude numbers the ring follows a path similar to a ring colliding with a flat surface, and only one surface depression, slightly outside of the ring, is formed. At higher Froude numbers the ring rebounds slightly due to the initial deformation of the surface, and waves are found to propagate outward in addition to a large depression outboard of the ring. The path of the vortex core lies farther away from the free surface than for the lower Froude number.

We have not modeled the second stage, the transition to three-dimensionality, here. The experiments suggest that it is sufficient to focus on only a short periodic segment of the vortex core at least as far as the vortex ring evolution is concerned. In this way, full simulations along the lines of Dommermuth and $\mathrm{Yue}^{7}$ could be used to address limited aspects of the evolution. The waves produced during the reconnection phase propagate both into the ring and outward and interact to form a complicated pattern. Similar waves were not observed in experiments using vortex pairs, see, e.g., Willmarth et al. ${ }^{3}$

\section{ACKNOWLEDGMENTS}

We would like to thank the members of the Program in Ship Hydrodynamics at the University of Michigan for their constructive discussions. We also express special thanks to Professor G. Meadows for his help in developing the experimental apparatus and the use of the towing tank.

This work was supported under the Program in Ship Hydrodynamics at the University of Michigan, funded by the University Research Initiative of the Office of Naval Research, Contract No. N000184-86-K-0684. The numerical simulations were performed on the computers at the San Diego Supercomputing Center, which is supported by the NSF.

IT. Sarpkaya, "Trailing-vortex wakes on the free surface," Proceedings of the 16th Symposium on Naval Hydrodynamics (National Academy, Washington, DC, 1987).

${ }^{2} \mathrm{~A}$. Hirsa, "An experimental investigation of vortex pair interaction with a clean and contaminated free surface," Ph.D. thesis, The University of Michigan, 1990.

${ }^{3}$ W. W. Willmarth, G. Tryggvason, A. Hirsa, and D. Yu, "Vortex pair generation and interaction with a free surface," Phys. Fluids A 1, 170 (1989).

${ }^{4}$ T. Sarpkaya, J. Elnitsky II, and R. E. Leeker, Jr., "Wake of a vortex pair on the free surface," Proceedings of the 17th Symposium on Naval Hydrodynamics (National Academy, Washington, DC, 1989).

${ }^{5} \mathrm{~J}$. G. Telste, "Potential flow about two counter-rotating vortices approaching a free surface," J. Fluid Mech. 201, 259 (1989).

${ }^{6} \mathrm{D}$. Yu and G. Tryggvason, "The free surface signature of unsteady, two-dimensional vortex flows," J. Fluid Mech. 218, 547 (1990).

${ }^{7}$ D. Dommermuth and D. Yue, "A numerical study of three-dimensional viscous interactions of vortices with a free surface," Proceedings of the 18th Symposium on Naval Hydrodynamics (National Academy, Washington, DC, 1991).

${ }^{8}$ A. W. Cerra, Jr. and C. R. Smith, "Experimental observations of vortex ring interaction with the fluid adjacent to a surface," Lehigh University Report FM-4, 1983. 
${ }^{9}$ W. J. A. Dahm, M. Scheil, and G. Tryggvason, "Dynamics of vortex interaction with a density interface," J. Fluid Mech. 205, 1 (1989).

${ }^{10} \mathrm{~L}$. P. Bernal and K. Madnia, "Interaction of a turbulent round jet with the free surface," in Ref. 4.

${ }^{11}$ L. P. Bernal and J. T. Kwon, "Vortex ring dynamics at a free surface," Phys. Fluids A 1, 449 (1989).

${ }^{12} \mathrm{~J}$. T. Kwon, "Experimental study of vortex ring interaction with a free surface," Ph.D. thesis, The University of Michigan, 1989.

${ }^{13}$ M. Song, N. Kachman, J. T. Kwon, L. P. Bernal, and G. Tryggvason, "Vortex interaction with a free surface," in Ref. 7.

${ }^{14} \mathrm{~N}$. Didden, "On the formation of vortex rings: Rolling-up and production of circulation," J. Appl. Math. Phys. (ZAMP) 30, 101 (1979).

${ }^{15} \mathrm{~A}$. Glezer, "The formation of vortex rings," Phys. Fluids 31, 3532 (1988).

${ }^{16}$ H. Lamb, Hydrodynamics (Dover, New York, 1945).

${ }^{17}$ D. M. Henderson and J. L. Hammack, "Experiments on ripple instabilities. Part 1. Resonant triads," J. Fluid Mech. 184, 15 (1987).

${ }^{18}$ L. P. Bernal, A. Hirsa, J. T. Kwon, and W. W. Willmarth, "On the interaction of vortex rings and pairs with a free surface for varying amounts of surface active agent," Phys. Fluids A 1, 2001 (1989).

${ }^{19}$ T. E. Brockett, M.-H. Kim, and J.-H. Park, "Limiting forms for surface singularity distribution when the field point is on the surface," $J$. Eng. Math. 23, 53 (1989).

${ }^{20}$ A. Leonard, "Vortex methods for flow simulation," J. Comput. Phys. 37, 289 (1980).

${ }^{21} \mathrm{P}$. G. Saffman, "The velocity of viscous vortex rings," Stud. Appl. Math. 4, 371 (1970).

${ }^{22} \mathrm{~T}$. Maxworthy, "The structure and stability of vortex rings," J. Fluid Mech. 51, 15 (1972).

${ }^{23}$ S. E. Widnall and J. P. Sullivan, "On the stability of vortex rings," Proc. R. Soc. London Ser. A 332, 465 (1973).

${ }^{24}$ S. C. Crow, "Stability theory for a pair of trailing vortices," AIAA J. 8, 2172 (1970).

${ }^{25}$ S. E. Widnall, D. B. Bliss, and C.-Y. Tsai, "The instability of short waves on a vortex ring," J. Fluid Mech. 66, 35 (1974).

${ }^{26} \mathrm{C}$.-Y. Tsai and S. E. Widnall, "The stability of short waves on a straight vortex filament in a weak externally imposed strain field," J. Fluid Mech. 73, 721 (1976). 